psychotic symptoms and a number of measures of school and family problems, including bullying, interparental domestic violence and physical and sexual abuse. ${ }^{2}$ We cited this in the paper. Furthermore, Kostic et al will be glad to know that a report on the relationship between childhood trauma and psychotic symptoms in another of the samples (study 2) is currently under review (details available from the authors on request). However, it is important to recognise that, again, the authors are raising an issue of causality in the relationship between psychotic symptoms and psychopathology; the point of the current paper, on the other hand, was to highlight new developments in our understanding of the importance of psychotic symptoms as clinical risk markers for psychopathology.

We appreciate that Kostic and colleagues are certainly not the only individuals who may have had conceptual misunderstandings about the above epidemiological points and we thank them for the opportunity to clarify some of these issues for the benefit of other readers with similar questions. We are also pleased to find that the Journal's readers are actively discussing the importance of assessing psychotic symptoms in the context of non-psychotic psychopathology. As well as recognising that psychotic symptoms are risk markers for a range of non-psychotic Axis I disorders in general, and for multimorbidity in particular, ${ }^{3}$ we would also especially encourage discussion about findings on the importance of these symptoms as risk markers for suicidal behaviour in young people with psychopathology. ${ }^{4}$ Considering the serious implications of these findings, an improved awareness of the significance of these symptoms among clinicians is urgently needed.

1 Kaufman J, Birmaher B, Brent D, Rao U, Ryan N. The Schedule for Affective Disorders and Schizophrenia for School Aged Children: Present and Lifetime Version. University of Pittsburgh, Western Psychiatric Institute and Clinic, 1996.

2 Kelleher I, Harley M, Lynch F, Arseneault L, Fitzpatrick C, Cannon M. Associations between childhood trauma, bullying and psychotic symptoms among a school-based adolescent sample. Br J Psychiatry 2008; 193: 378-82.

3 Kelleher I, Keeley H, Corcoran P, Lynch F, Fitzpatrick C, Devlin N, et al. Clinicopathological significance of psychotic experiences in non-psychotic young people: evidence from four population-based studies. $\mathrm{Br} J$ Psychiatry 2012; 201: 26-32.

4 Kelleher I, Lynch F, Harley M, Molloy C, Roddy S, Fitzpatrick C, et al. Psychotic symptoms in adolescence index risk for suicidal behavior: findings from two population-based case-control clinical interview studies. Arch Gen Psychiatry 2012; doi: 10.1001/archgenpsychiatry.2012.164. (Epub ahead of print.)

Ian Kelleher, Department of Psychiatry, Royal College of Surgeons in Ireland Education and Research Centre, Beaumont Hospital, Dublin 9, Ireland. Email: iankelleher@rcsi.ie; Mary Cannon, Department of Psychiatry, Royal College of Surgeons in Ireland, and Department of Psychiatry, Beaumont Hospital, Dublin, Ireland

doi: 10.1192/bjp.202.2.152a

\section{The need for inclusion of concepts of recovery in clinical trials}

The study by Tohen and colleagues addresses a field of clinical practice that has traditionally posed a great deal of therapeutic challenge. ${ }^{1}$ Evidence of potential therapeutic response in initial trials are therefore welcome and the authors are right to call for further research to assess the efficacy of olanzapine, while cautioning in relation to the high non-adherence rates observed with this medication.

The authors also attempt to explore the degree of recovery experienced by individuals within their trial. It is correct that this concept is addressed, even in early trials such as this. By considering concepts such as recovery, clinical trials can provide information that allows clinicians and service users to make truly informed decisions in relation to treatment options. Calls for the inclusion of recovery-oriented outcomes in clinical trials into various disorders have been made. ${ }^{2,3}$

However, in this study the authors appear to make the mistake of conflating the concepts of recovery and symptom remission. The concept of recovery is generally recognised as being more than simple remission of symptoms, instead involving a deeper acceptance of disorder and personal adaptation to experience. In this journal, a narrative review by Leamy et al described five main themes of recovery that are representative of this concept; they are the sense of: connectedness, hope, identity, meaning and empowerment. ${ }^{4}$

Measures such as the Montgomery-Åsberg Depression Rating Scale (MADRS) are valuable in their sensitive detection of change in the symptoms of depressive disorders but they do not address the core concepts of recovery. ${ }^{5}$ Simple definition of recovery as a sustained period of symptom remission (MADRS $\geqslant 12$ for $\leqslant 4$ weeks) as in this paper is therefore inadequate.

The development of suitable recovery-oriented outcome measures for inclusion in clinical trials is urgently required to allow us to develop an evidence base that considers all aspects of treatment and allows us to provide service users with the information they require to make informed treatment decisions.

1 Tohen M, McDonnell DP, Case M, Kanba S, Ha K, Fang YR, et al. Randomised, double-blind, placebo-controlled study of olanzapine in patients with bipolar I depression. Br J Psychiatry 2012; 201: 376-82.

2 Slade $M$, Hayward M. Recovery, psychosis and psychiatry: research is better than rhetoric. Acta Psychiatr Scand 2007; 116: 81-3.

3 Bateman AW. Treating borderline personality disorder in clinical practice. Am J Psychiatry 2012; 169: 560-3.

4 Leamy M, Bird V, LeBoutillier C, Williams J, Slade M. Conceptual framework for personal recovery in mental health: systematic review and narrative synthesis. Br J Psychiatry 2011; 199: 445-52.

5 Montgomery SA, Åsberg M. A new depression scale designed to be sensitive to change. Br J Psychiatry 1979; 134: 382-89.

Andrew Shepherd, CT3 Psychiatry Trainee, Greater Manchester West Mental Health Foundation Trust, UK. Email: shrinking81@gmail.com

doi: 10.1192/bjp.202.2.153

Author's reply: I agree with Dr Shepherd that there is a need to better define outcomes in clinical trials. It is correct that we defined recovery as a sustained remission of psychiatric symptoms. Indeed, we followed the definition recommended by the International Society for Bipolar Disorders (ISBD). ${ }^{1}$ The term recovery in the ISBD consensus guidelines is based on sustained absence of or low-severity symptomatology without considering functional outcomes.

Observational studies in bipolar disorder, however, have in fact shown that symptomatic remission is not always accompanied by functional recovery, ${ }^{2,3}$ which supports Dr Shepherd's point that symptom resolution is not always followed by improved functional outcomes such as adaptation to the experience.

I agree with Dr Shepherd that functional outcomes allow clinicians to make better treatment decisions that are more patient-centred. Furthermore, in the consideration of regulatory approval around the globe, symptom improvement is the main criterion for a new treatment to get approved. Including functional outcomes in the regulatory approval of pharmacological treatments would be beneficial to patients.

1 Tohen M, Frank E, Bowden CL, Colom F, Ghaemi NS, Yatham LN, et al. The International Society for Bipolar Disorders (ISBD) Task Force report on the 
nomenclature of course and outcome in bipolar disorders. Bipolar Disord 2009; 11: 453-73.

2 Tohen M, Waternaux CM, Tsuang MT. Outcome in mania. A 4-year prospective follow-up of 75 patients utilizing survival analysis. Arch Gen Psychiatry 1990; 47: 1106-11

3 Tohen M, Zarate CA, Hennen J, Khalsa HM, Strakowski SM, Gebre-Medhin P, et al. The McLean-Harvard First-Episode Mania Study: prediction of recovery and first recurrence. Am J Psychiatry 2003; 160: 2099-107.

Mauricio Tohen, Department of Psychiatry, University of New Mexico Health Science Center, Albuquerque, New Mexico, USA. Email: tohen@uthscsa.edu

doi: $10.1192 /$ bjp.202.2.153a

\section{Is this a non-inferiority trial?}

Crawford et al ${ }^{1}$ have set out to investigate whether screening for suicidal ideation among people who attend primary care services and have signs of depression increases the short-term incidence of feeling that life is not worth living. It seems to me that this is a non-inferiority trial, i.e. the authors want to show that screening is no worse than not screening. This raises a number of design issues.

First, the trial is powered to detect an increase in the proportion who felt their life was not worth living from $30 \%$ to $45 \%$. This seems a clinically large increase and suggests that anything short of a $50 \%$ increase in relative risk is acceptable. Second, having calculated the sample size based on relative risk, they analyse the main results using odds ratios rather than relative risk, so that it is difficult to see what sort of increase in relative risk was found and impossible to see the confidence interval around the relative risk. Third, if this is seen as a non-inferiority trial, arguably screening would be regarded as non-inferior provided that the possibility of the suicidal ideation rate being $50 \%$ worse than non-screening could be ruled out (in the sense that the $95 \%$ confidence interval for the difference in ideation rates would not include $50 \%$ inferiority relative to the non-screened group). ${ }^{2}$ We only have the confidence interval around the odds ratio to go on, but given how wide that is, it is highly likely that the confidence interval would include the $50 \%$ increase in relative risk. For these reasons I think the results should be treated with caution.

1 Crawford MJ, Thana L, Methuen C, Ghosh P, Stanley SV, Ross J, et al. Impact of screening for risk of suicide: randomised controlled trial. $\mathrm{Br} J$ Psychiatry 2011; 198: 379-84.

2 Pocock SJ. The pros and cons of noninferiority trials. Fund Clin Pharmacol 2003; 17: 483-90.

\section{Peter O'Halloran, Lecturer, Queen's University Belfast, UK. Email:}

p.ohalloran@qub.ac.uk

doi: 10.1192/bjp.202.2.154

Authors' reply: Peter O'Halloran raises a good point about this study: it was underpowered. Even slightly higher levels of suicidal thinking among people who are screened for suicide risk would be clinically important and our study was only powered to detect a fairly large difference.

However, at the start of the study we faced a dilemma; a sizeable minority of local general practitioners told us that they were uncomfortable asking people with depression about suicidal thoughts because they were concerned that this could 'make them feel suicidal. ${ }^{1}$ Subsequent discussions with medical students and trainee psychiatrists (and indeed members of the ethics committee that reviewed the study protocol) revealed that these concerns were shared by others. A sample size calculation based on a non-inferiority hypothesis and using a smaller but still clinically important difference in levels of suicidal thinking would have required a sample size several times larger than the one we recruited. As Norman and colleagues have recently pointed out, ${ }^{2}$ sample size calculations are usually a compromise between statistical considerations, economics and logistical constraints. When we embarked on the study we knew that we did not have the resources to recruit a sample large enough to detect a small difference in levels of suicidal thinking among those who were and were not screened. However, we hoped that we could rule out the possibility of a large difference and this is therefore what we set out to do. The 95\% confidence intervals around the odds ratio for the likelihood of suicidal thoughts among those that were screened were broad (0.66-1.18) and are compatible with either higher or lower levels of suicidal thinking in those who are screened compared with those who are not. Despite this limitation, these are the first data that test the veracity of a belief that was held by many and may have been an obstacle to screening for risk of self-harm in this high-risk group.

As we pointed out in the Discussion of our paper, it was not possible to collect data needed to calculate relative risks associated with screening because this would have meant collecting baseline levels of suicidal ideation from all those in the study. This would have exposed those in the control arm of the study to the very factor that the study was designed to examine.

1 Bajaj P, Borreani E, Ghosh P, Methuen C, Patel M, Crawford MJ. Screening for suicidal thoughts in primary care: the views of patients and general practitioners. Ment Health Fam Med 2008; 4: 229-35.

2 Norman G, Monterio S, Salama S. Sample size calculations: should the emperor's clothes be off the peg or made to measure? BMJ 2012; 345: e5278.

Mike J. Crawford, Centre for Mental Health, Faculty of Medicine, Imperial College London, Claybrook Centre, 37 Claybrook Road, London W6 8LN, UK. Email: m.crawford@imperial.ac.uk; Priya Bajaj, Central and North West London NHS Foundation Trust, UK.

doi: 10.1192/bjp.202.2.154a

\section{Delay in starting clozapine and treatment guidelines}

There is a reasonable level of information to suggest that clozapine is effective in patients who have treatment-resistant schizophrenia. Hence, clozapine should be started at the right time so that patients can draw maximum benefit from it. In this vein, the article by Howes et $a l^{1}$ provides important insights into the clinical practice with regard to the use of clozapine. The authors showed that clozapine is delayed by about 4 years and many patients are treated with polypharmacy and receive higher than recommended doses, which is contrary to the recommendations made by several practice guidelines. However, it is important to note that the conclusions drawn about the delay in starting clozapine might not be a true reflection of actual delay, because often patients who are offered clozapine refuse to take it. Hence, some of the delay may be due to lack of agreement of the patient and this in general does not reflect the delay in the clinician offering the medication. It would have been better had the authors extracted the data pertaining to initial offering of clozapine and the number of patients who refused clozapine at the first instance as part of this study. This would have actually given the true clinical picture.

Another issue is the definition of duration of illness used. The authors have defined duration of illness as 'the time from the first recording of the diagnosis of a psychotic illness by a clinician to the present', which may not be a true reflection of duration of 